Supporting Information

\title{
Effects of Impurities on the Thermal and Electrical Transport Properties of Cubic Boron Arsenide
}

Xi Chen, ${ }^{1 *}$ Chunhua $\mathrm{Li}^{2}{ }^{2}$ Youming Xu, ${ }^{1}$ Andrei Dolocan, ${ }^{3}$ Gareth Seward, ${ }^{4}$ Ambroise Van Roekeghem, ${ }^{5}$ Fei Tian, ${ }^{6}$ Jie Xing, ${ }^{7}$ Shucheng Guo, ${ }^{1}$ Ni Ni, ${ }^{7}$ Zhifeng Ren, ${ }^{6}$ Jianshi Zhou, ${ }^{3}$ Natalio Mingo, ${ }^{5}$ David Broido, ${ }^{2}$ and $\mathrm{Li} \mathrm{Shi}^{3,8}$

${ }^{1}$ Department of Electrical and Computer Engineering, University of California, Riverside, California 92521, USA

${ }^{2}$ Department of Physics, Boston College, Chestnut Hill, Massachusetts 02467, USA

${ }^{3}$ Materials Science and Engineering Program, Texas Materials Institute, The University of Texas at Austin, Austin, Texas 78712, USA

${ }^{4}$ Earth Science, University of California, Santa Barbara, California 93106, USA

${ }^{5}$ Université Grenoble Alpes, CEA, LITEN, 17 rue des Martyrs, 38054 Grenoble Cedex 9, France

${ }^{6}$ Department of Physics and the Texas Center for Superconductivity, University of Houston, Houston, Texas 77204, USA

${ }^{7}$ Department of Physics and Astronomy and California NanoSystems Institute, University of California, Los Angeles, California 90095, USA

${ }^{8}$ Walker Department of Mechanical Engineering, The University of Texas at Austin, Austin, Texas 78712, USA

*E-mail: xichen@ucr.edu 


\section{Hall measurements}

The measured Hall resistivity data as a function of magnetic field for four BAs samples are shown in Figure S1. All the BAs samples exhibit $p$-type conducting behavior.

(a)

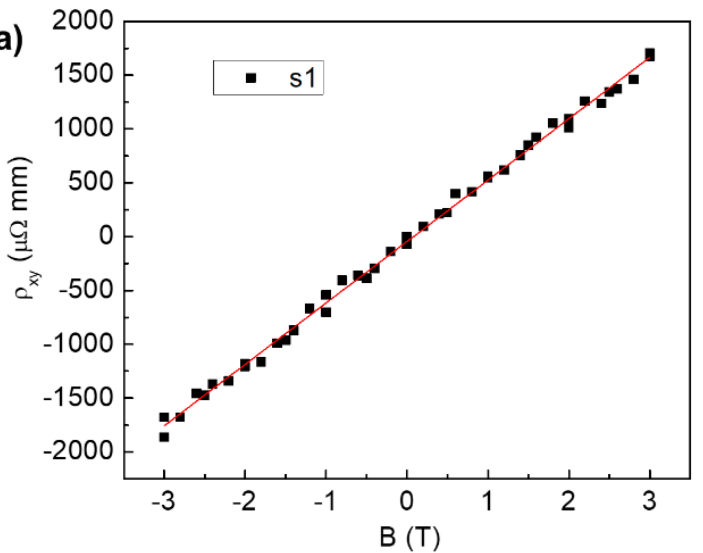

(c)

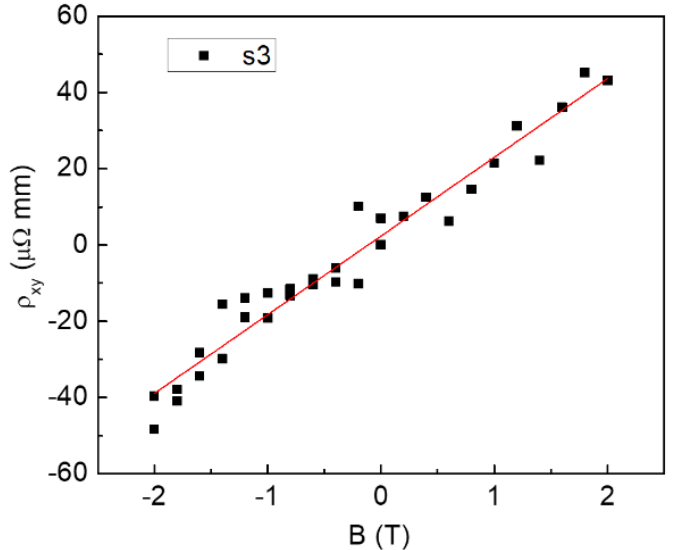

(b)
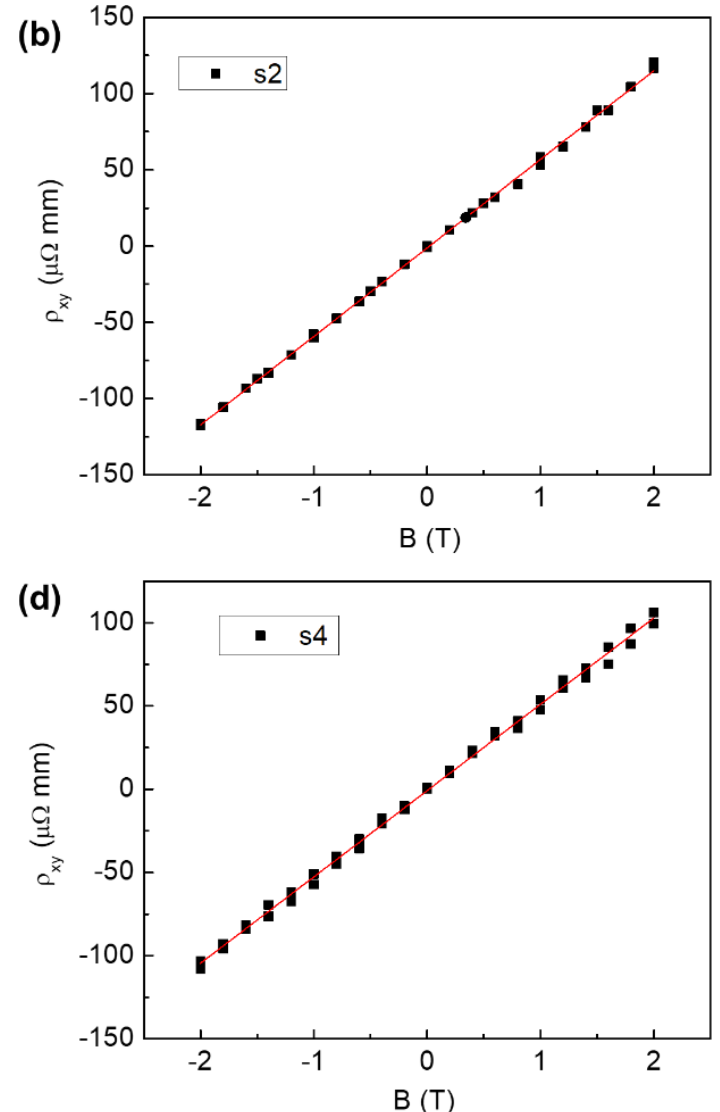

Fig. S1. Measured Hall resistivity of four BAs samples.

\section{Time-of-flight secondary ion mass spectrometry (TOF-SIMS) measurements}

Figure S2 shows additional TOF-SIMS measurement results of the BAs samples. The impurities are identified to be chemically bound to the boron or arsenic atoms since $\mathrm{BH}^{-}, \mathrm{BAsO}^{-}, \mathrm{CB}^{-}, \mathrm{SiAs}^{-}$, and $\mathrm{TeAs}^{-}$clusters are detected in the TOF-SIMS data. Other impurities such as $\mathrm{S}$ and $\mathrm{Cu}$ are also present in both samples. 

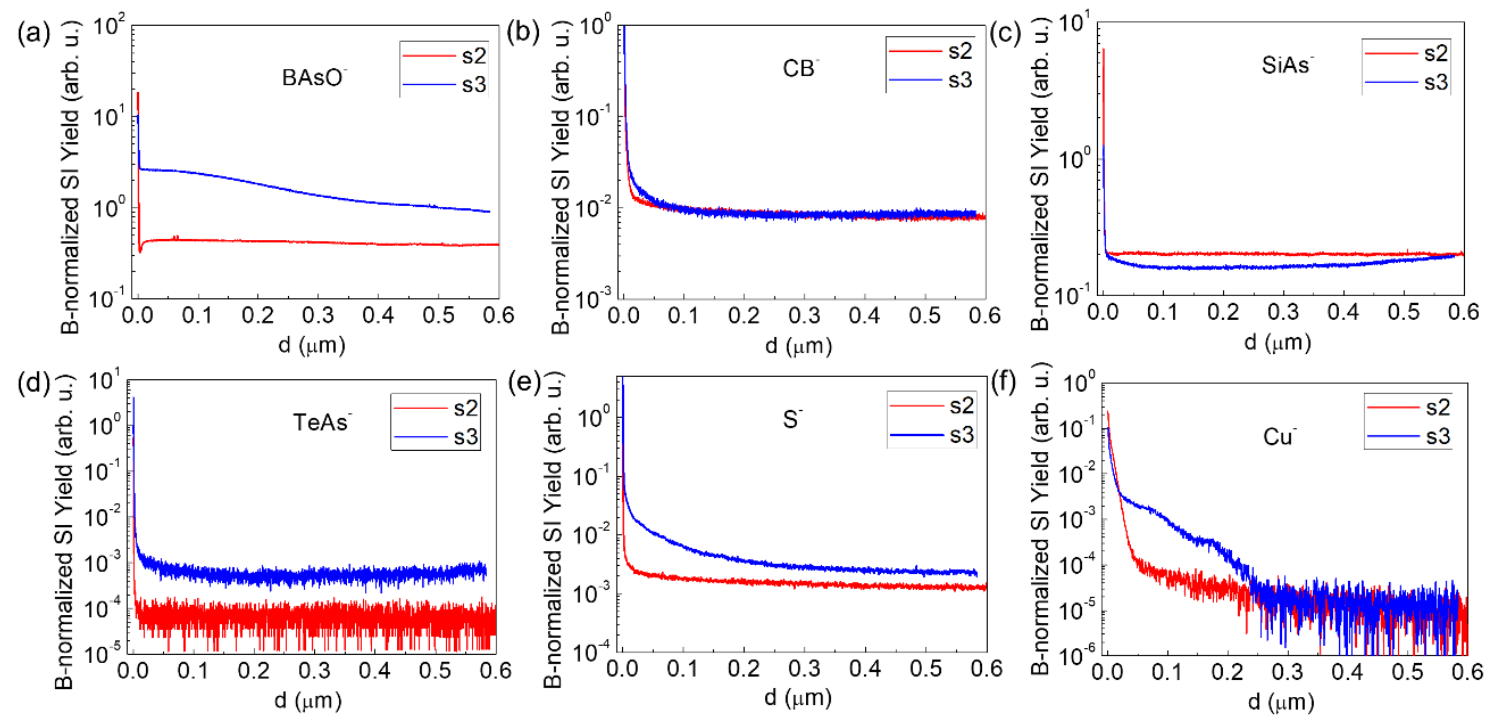

Fig. S2. Characterization of impurities in the s2 and s3 samples by TOF-SIMS.

\section{Energy-dispersive X-ray (EDX) measurement}

Figure S3 shows the backscattered scanning electron microscope (SEM) image of the s3 sample. The white regions are found to be the Te-rich phase based on the energy-dispersive X-ray (EDX) analysis, as shown in Figure S4. The atomic ratio in the white region is found to be Te : As : Si : $\mathrm{Ag}=50.8: 44.9: 3.4: 0.9$.

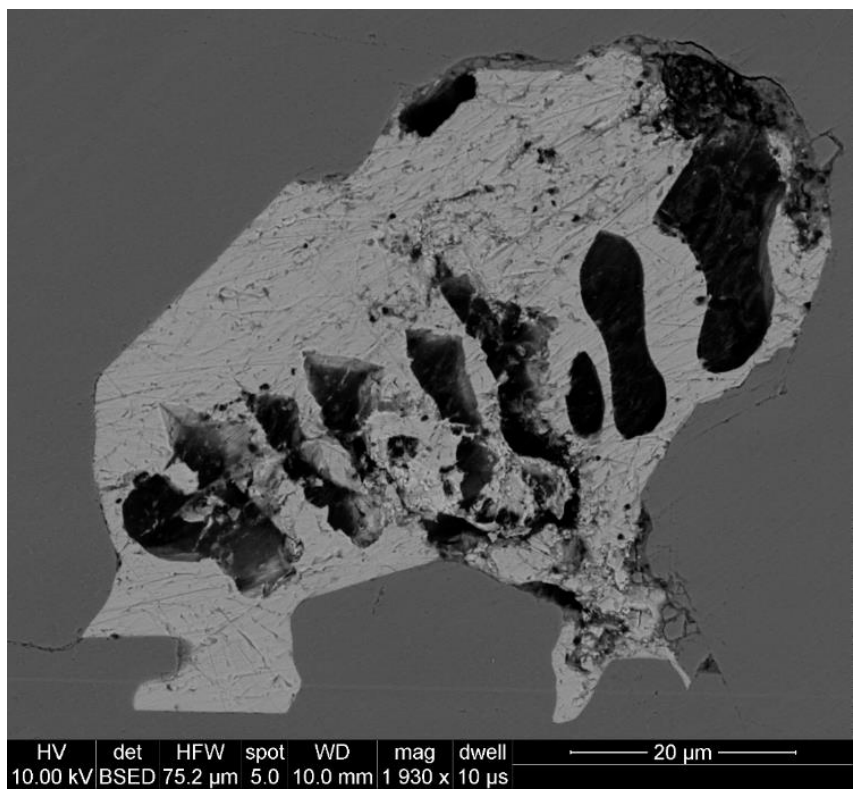

Fig. S3. Backscattered scanning electron micrograph of the s3 sample. 


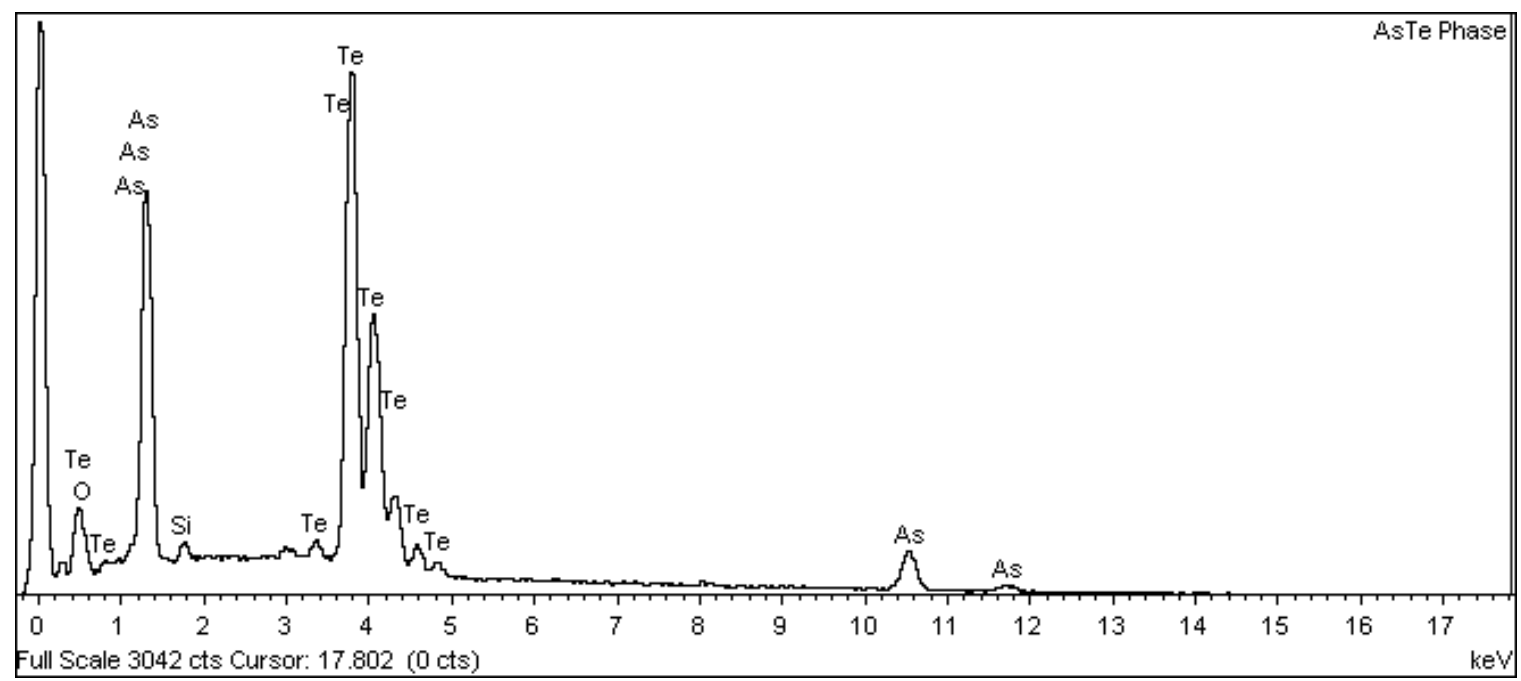

Fig. S4. EDX result of the white region in Fig. S3.

\section{Single parabolic band (SPB) model}

In the SPB model, the Seebeck coefficient $(S)$ is given by ${ }^{[1]}$

$$
\begin{aligned}
& S=\frac{k_{B}}{e}\left(\frac{\left(\frac{5}{2}+r\right) F_{r+\frac{3}{2}}(\eta)}{\left(\frac{3}{2}+r\right) F_{r+\frac{1}{2}}(\eta)}-\eta\right) \\
& F_{i}(\eta)=\int_{0}^{\infty} y^{i}\left(e^{y-\eta}+1\right)^{-1} d y \\
& \eta=\left(E_{F}-E_{V}\right) /\left(k_{B} T\right)
\end{aligned}
$$

Where $k_{\mathrm{B}}$ is the Boltzmann constant, $e$ is the electron charge, and $r$ is the scattering parameter, which equals to $-1 / 2$ and $3 / 2$ for acoustic phonon scattering and ionized impurity scattering, respectively. Based on the temperature dependence of the mobility data, we find that both acoustic phonon and ionized impurity scattering are important in BAs. Therefore, $r$ takes a value of one for the mixed scattering. ${ }^{[2]}$ The Fermi integral, $F_{i}(\eta)$, is a function of the reduced chemical potential $\eta$, which is related to the chemical potential $\left(E_{\mathrm{F}}\right)$ and the energy of the top of the valence band $\left(E_{\mathrm{v}}\right)$. Both $E_{\mathrm{F}}$ and $E_{\mathrm{v}}$ are measured from the conduction band edge, and $\eta$ is negative when $E_{\mathrm{F}}$ is in the gap. The Hall carrier concentration can be expressed as 


$$
\begin{gathered}
p_{H}=4 \pi\left(\frac{2 m^{*} k_{B} T}{h^{2}}\right)^{3 / 2} \frac{F_{1 / 2}(\eta)}{r_{H}} \\
r_{H}=\frac{3(2 r+3 / 2)}{2(r+3 / 2)^{2}} \frac{F_{2 r+1 / 2}(\eta) F_{-1 / 2}(\eta)}{F_{r+1 / 2}^{2}(\eta)}
\end{gathered}
$$

where $\hbar$ is the reduced Plank constant, $m^{*}$ is the density-of-states effective (DOS) mass accounting for multiple valleys, and $r_{\mathrm{H}}$ is the Hall factor.

\section{Schematic of displaced $O_{A s}$ defect}

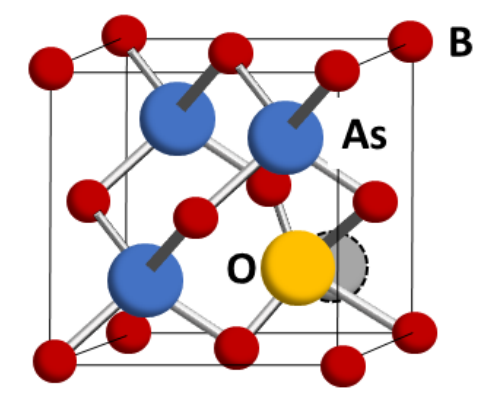

Fig. S5. Crystal structure of O-doped BAs showing the large displacement of $\mathrm{O}_{\mathrm{As}}$ impurity. The dashed circle presents the original position of As.

\section{References}

[1] Liu, W.; Chi, H.; Sun, H., Zhang, Q.; Yin, K.; Tang, X.; Zhang, Q.; Uher, C. Advanced thermoelectrics governed by a single parabolic band: $\mathrm{Mg}_{2} \mathrm{Si}_{0.3} \mathrm{Sn}_{0.7}$, a canonical example. Phys. Chem. Chem. Phys. 2014, 16, 6893.

[2] Mao, J.; Shuai, J.; Song, S.; Wu, Y.; Dally, R.; Zhou, J.; Liu, Z.; Sun, J.; Zhang, Q.; dela Cruz, C.; et al. Manipulation of ionized impurity scattering for achieving high thermoelectric performance in n-type $\mathrm{Mg}_{3} \mathrm{Sb}_{2}$-based materials. Proc. Natl. Acad. Sci. U.S.A. 2017, 114, 10548 . 